\title{
ATUAÇÃO DO FARMACÊUTICO NA ASSISTÊNCIA A SAÚDE EM FARMÁCIAS COMUNITÁRIAS
}

\author{
PERFORMANCE OF THE PHARMACIST IN HEALTH CARE IN COMMUNITY \\ PHARMACIES
}

\author{
Cristina França ${ }^{1}$ \\ Leonardo Guimarães de Andrade ${ }^{2}$
}

RESUMO: Farmácia comunitária é o estabelecimento farmacêutico que presta atendimento primário à população, sob responsabilidade técnica de um farmacêutico. Nos últimos anos vem ocorrendo um movimento no Brasil em busca da ampliação da atuação do farmacêutico e da participação da farmácia comunitária no sistema de saúde brasileiro com o desenvolvimento de serviços farmacêuticos clínicos. Para atender essa necessidade há uma mudança no perfil do profissional farmacêutico que resultou na atualização da definição de farmácia e na publicação das RDCs do CFF no 585 e 586 que regulamentam as atribuições clínicas do farmacêutico e a prescrição farmacêutica.

Palavras-chave: Farmácia Comunitária. Assistência a Saúde. Atenção Farmacêutica.

ABSTRACT: Community pharmacy is the pharmaceutical establishment that provides primary care to the population, under the technical responsibility of a pharmacist. In recent years, there has been a movement in Brazil seeking to expand the role of the pharmacist and the participation of community pharmacy in the Brazilian health system with the development of clinical pharmaceutical services. To meet this need, there is a change in the profile of the pharmacist that resulted in the updating of the definition of pharmacy and the publication of the RDCs of CFF No. 585 and 586 that regulate the clinical attributions of the pharmacist and the pharmaceutical prescription.

Keywords: Community pharmacy. Health Care. Pharmaceutical attention.

\section{INTRODUÇÃO}

No início da profissão, os farmacêuticos atuavam nas boticas, constituídos por estabelecimentos pequenos familiar onde pesquisas, manipulações e novos produtos eram avaliados, no qual uma grande parte tinha origem animal ou vegetal, pois a

\footnotetext{
I Graduação em Farmácia pela UNIG - Universidade Iguaçu- Nova Iguaçu- RJ. E-mail: cris_pretar6@hotmail.com.

${ }^{2}$ Professor e orientador dos discentes do curso de farmácia pela UNIG.
} 
função era ter como garantia que os medicamentos fossem puros e não sofressem alterações durante o seu preparo de acordo com as técnicas daquela época. Porém com a grande industrialização de medicamentos um novo rumo surgiu da profissão, apresentou-se uma especialização do profissional farmacêutico, onde ele estaria participando ativamente em tudo que dizia respeito a medicamentos, desde a prescrição, transcrição, dispensação, administração e primordialmente o acompanhamento do paciente junto a equipe de saúde, podendo então desenvolver suas habilidades relacionadas a farmacoterapia (BRASIL, 2013).

A farmácia tem fundamental importância como porta de acesso da população em relação ao consumo de medicamentos e devia ser entendida como um posto avançado de atenção primária de saúde. Segundo dados do Ministério da Fazenda, as farmácias e drogarias seriam responsáveis por $76 \%$ do fornecimento direto de medicamentos à população (BRASIL, 2013).

O termo "farmácia comunitária" refere-se aos estabelecimentos farmacêuticos não hospitalares e não ambulatoriais que atendem à comunidade. As farmácias comunitárias no Brasil são, em sua maioria, privadas, de propriedade particular, mas existem também farmácias públicas, sejam elas vinculadas à rede nacional de farmácias populares ou às esferas públicas municipais ou estaduais (BAREATA, 2003).

Farmácias comunitárias referem-se aos estabelecimentos do comércio varejista privado tendo o farmacêutico como responsável técnico, atendendo às exigências da Lei no 5.991/73 do Ministério da Saúde. É necessário destacar que, neste trabalho, o termo, farmácia comunitária exclui as farmácias de manipulação e as farmácias públicas, referindo-se tão somente às farmácias ditas comerciais e drogarias. Nestas, o atendimento ao paciente acontece no nível de atenção primária à saúde, com a responsabilidade técnica, legal e privativa de farmacêutico (BRASIL, 1973).

Há, no Brasil, também, uma distinção legal entre farmácia e drogaria, sendo estas últimas proibidas de atuar na manipulação ou no fornecimento fracionado de medicamentos. A drogaria é definida como "estabelecimento de dispensação e comércio de drogas, medicamentos, insumos farmacêuticos e correlatos em suas embalagens originais" (BRASIL, 2013). 
A atualização das definições ocorreu em 2014, na lei 13.021 que define farmácia como:

unidade de prestação de serviços destinada a prestar assistência farmacêutica, assistência à saúde e orientação sanitária individual e coletiva, na qual se processe a manipulação e/ou dispensação de medicamentos magistrais, oficinais, farmacopeicos ou industrializados, cosméticos, insumos farmacêuticos, produtos farmacêuticos e correlatos (BRASIL, 2014).

De acordo com essa resolução as farmácias podem ser classificadas em "farmácia com manipulação" ou em "farmácia sem manipulação ou drogaria”. Ambas as leis exigem a presença do farmacêutico no estabelecimento em todo o horário de funcionamento (Brasil, 1973; Brasil, 2014).

Com essa atualização da definição de farmácia esse estabelecimento deixou de ser apenas comercial e tornou-se um local de prestação de assistência farmacêutica e assistência à saúde. Nesse contexto, em 2013 foram publicadas as resoluções do Conselho Federal de Farmácia (CFF) no 585 e 586. A primeira regulamenta as atribuições clínicas do farmacêutico e a segunda regula a prescrição farmacêutica (BRASIL, 2013a; BRASIL, 2013b). Ambas visam à "promoção, proteção e recuperação da saúde" (BRASIL, 2013a).

\section{OBJETIVOS:}

\section{I Objetivo Geral:}

Identificar à importância do Farmacêutico na atuação a assistência a saúde em farmácias comunitárias.

\subsection{Objetivos Específicos:}

- Relatar o que é Assistência Farmacêutica;

- Identificar os serviços que o a Farmácia Comunitária deve prestar;

- Mencionar a importância do serviço de acompanhamento farmacêutico;

- Relatar a realidade da farmácia comunitária no Brasil;

- Demonstrar a estrutura de uma farmácia comunitária.

\section{METODOLOGIA}


Para atingir o objetivo desse trabalho optou-se por uma pesquisa bibliográfica, baseando em artigos publicados no período de 2013 a 2019. O levantamento bibliográfico ocorreu na Biblioteca Virtual em Saúde, na base de dados caracterizados como Literatura Latino - Americana em Ciências de Saúde (LILACS) e na Scientifc Electronic Library Online (SciELO). Para o levantamento do material, foram utilizados os Descritores: Farmácia Comunitária; Assistência a Saúde; Atenção Farmacêutica.

Também foram pesquisadas teses, dissertações e livros publicados nesse período, no sentido de aprofundar aspectos relacionados ao tema. No desenvolvimento do trabalho, os principais passos seguidos foram a leitura e a análise de vários autores, com a realização de fichamentos e a interpretação dos autores sobre o assunto.

Quanto à formatação e a configuração a presente pesquisa visará seguir as normas da ABNT (Associação Brasileira de Normas Técnicas).

\section{JUSTIFICATIVA}

O último contato entre um paciente e um profissional de saúde, será em uma farmácia, onde a atuação do farmacêutico terá grande importância para a promoção à saúde, bem como o uso racional de medicamentos. Esse momento é a oportunidade do farmacêutico de fazer com que a farmácia não seja um simples comércio e sim, um estabelecimento de saúde. Portanto, esse trabalho tem sua justificativa pautada na necessidade de aprimoramento continuado dos profissionais de farmácia no desempenho de suas atividades.

\section{REVISÃO DA LITERATURA 5.I ASSISTÊNCIAS FARMACÊUTICAS}

Uma das áreas de atuação do farmacêutico é na farmácia comunitária e dentre as atividades desenvolvidas está a assistência farmacêutica. De acordo com a Lei 13.02I de 2014 a Assistência Farmacêutica (ASF) é: Conjunto de ações e de serviços que visem a assegurar a assistência terapêutica integral e a promoção, a proteção e a recuperação 
da saúde nos estabelecimentos públicos e privados que desempenhem atividades farmacêuticas, tendo o medicamento como insumo essencial e visando ao seu acesso e ao seu uso racional (BRASIL, 2014).

Portanto, o objetivo da assistência farmacêutica é oferecer aos pacientes informações necessárias sobre a doença, uso correto, seguro e racional dos medicamentos e propostas terapêuticas que integradas com outras práticas de atenção à saúde contribuem decisivamente para melhoria da qualidade desta atenção (CONSELHO REGIONAL DE FARMÁCIA DO ESTADO DE SÃO PAULO, 2010).

A ASF envolve seleção, programação, aquisição, distribuição, dispensação, garantia da qualidade dos produtos e serviços, acompanhamento e avaliação de sua utilização com o objetivo de melhorar a qualidade de vida da população (CONSELHO NACIONAL DE SAÚDE, 2004).

Além de desenvolver este conjunto de atividades, o profissional farmacêutico presta contínua promoção de saúde, uma vez que, adota a clínica para restabelecer a saúde dos usuários, sendo o responsável pela comunicação com o paciente, ou seja, desenvolve atenção primária à saúde, desta forma, irá aumentar a qualidade do atendimento e consequentemente a relação entre eles será fortalecida (BASTOS; CAETANO, 2010; CONSELHO FEDERAL DE FARMÁCIA, 2009; GALATO et. al., 2008, SOUZA, 2012).

A atenção farmacêutica é uma atividade que pode ser oferecida nas farmácias comunitárias. Várias publicações apontam que os serviços de atenção primária contribuem para diminuição da internação de pacientes, no Reino Unido, por exemplo, a Sociedade Farmacêutica, tem trabalhado junto ao Ministério da Saúde do

país, em virtude do aumento dos cuidados e serviços farmacêuticos prestados nas farmácias (CONSELHO REGIONAL DE FARMÁCIA DO ESTADO DE SÃO PAULO, 2010).

Segundo um estudo realizado por DOBLINSKI e colaboradores (2012), a população necessita de mais informações no momento do atendimento nas farmácias, 
pois na maioria das vezes, o diálogo com o médico nem sempre é completo, desta forma, o profissional farmacêutico é o responsável por prestar a atenção farmacêutica, garantindo a eficácia terapêutica do tratamento da doença e zelando pela manutenção da saúde e qualidade de vida do paciente.

As pessoas devem ser conscientizadas quanto ao uso indiscriminado de medicamentos, sem orientação médica, que se elevam os riscos de reações adversas e no agravamento de doenças. Portanto, a assistência farmacêutica garante a melhoria da qualidade e a eficácia do fármaco, na orientação do paciente para que utilize de forma correta, aumentando-se a aderência terapêutica prescrita e na prevenção de efeitos colaterais ou na interação de fármacos, sendo um fator fundamental para que pacientes recebam cuidados na administração de fármacos e ferramenta estratégica na manutenção da saúde da população brasileira (FERNANDES; CEMBRANELLI, 2015).

\subsection{SERVIÇOS FARMACÊUTICOS DESCRITOS NA RDC 499/o8}

Os serviços farmacêuticos não podem ser desprezados pela população e as farmácias que não prestam esses serviços estão sendo omissas e irresponsáveis com seus clientes, devendo ser penalizadas (LOPES JÚNIOR, 2013). O profissional é o responsável por todos os serviços farmacêuticos de atenção à saúde que são prestados aos pacientes (CONSELHO FEDERAL DE FARMÁCIA, 202I).

ARDC 499/2008 do Conselho Federal de Farmácia destaca os serviços que podem ser oferecidos em uma farmácia comunitária, conforme a tabela I. Após a prestação dos serviços farmacêuticos, deve-se registrar em um formulário próprio quais serviços foram desenvolvidos. A via original do formulário deve ficar arquivada na farmácia, uma cópia entregue ao usuário e as demais encaminhadas aos profissionais de saúde, se necessário (CONSELHO FEDERAL DE FARMÁCIA, 202I). 
Tabela I: Serviços farmacêuticos descritos na RDC 499/2008.

\begin{tabular}{|c|c|}
\hline Serviços farmacêuticos & Descrição \\
\hline $\begin{array}{c}\text { Determinação dos parâmetros } \\
\text { bioquímicos }\end{array}$ & $\begin{array}{l}\text { Deve ser utilizada apenas para prevenção de enfermidades } \\
\text { e monitoramento do tratamento, sendo quantificado teor } \\
\text { sanguineo de glicose, colesterol total e triglicérides. Estes } \\
\text { testes devem ser registrados (protocolos) e não devem ser } \\
\text { fornecidos como diagnóstico. No caso de qualquer } \\
\text { alteração, o usuário deve procurar assistência médica. }\end{array}$ \\
\hline Verifícação d & $\begin{array}{l}\text { É outro serviço farmacêutico prestado com intuito de } \\
\text { prevenir enfermidades ou monitorar tratamento, uma vez } \\
\text { que, os resultados não podem ser fornecidos como } \\
\text { diagnóstico clinico, nem serem utilizados para prescrição } \\
\text { de medicamentos. Devem ser registrados em ficha e/ou } \\
\text { carteira de hipertenso do usuário. }\end{array}$ \\
\hline $\begin{array}{c}\text { Verificação da temperatura } \\
\text { corporal }\end{array}$ & $\begin{array}{l}\text { É um serviço facultado ao farmacêutico, com o intuito de } \\
\text { prevenir enfermidades ou monitorar o tratamento } \\
\text { farmacológico. Na observação de qualquer alteração da } \\
\text { temperatura corporal, o usuário poderá ser aconselhado a } \\
\text { procurar a devida assistência médica. }\end{array}$ \\
\hline Colocação de brincos & $\begin{array}{l}\text { Atividade que pode ser oferecida pelo farmacêutico, } \\
\text { porém para realização deste procedimento, os brincos } \\
\text { devem ser estéreis, devidamente acondicionados em } \\
\text { embalagens, com a finalidade de promover proteção do } \\
\text { usuário. }\end{array}$ \\
\hline $\begin{array}{c}\text { Assistência farmacêutica } \\
\text { domiciliar }\end{array}$ & $\begin{array}{l}\text { A assistência farmacêutica domiciliar é outro serviço que } \\
\text { pode ser oferecido pelo profissional farmacêutico, } \\
\text { possibilitando melhoria do acesso dos pacientes e da } \\
\text { população aos cuidados farmacêuticos. }\end{array}$ \\
\hline
\end{tabular}


Esse procedimento pode ser realizado nas farmácias comunitárias, quando não há presença de hemorragia arterial e quando não for necessário fazer sutura. Sendo proibida a realização de lavagem ou curativo na região do Curativos de pequeno porte ouvido. O profissional também não deve fazer retirada de pontos, nem atender pacientes com infecção profunda ou com mordidas de animais, pois estes pacientes devem procurar um atendimento hospitalar ou ambulatorial

\begin{tabular}{|c|c|}
\hline $\begin{array}{l}\text { pação em campa } \\
\text { de saúde }\end{array}$ & $\begin{array}{l}\text { As farmácias e drogarias poderão participar de } \\
\text { campanhas, sobre promoção e proteção de saúde, } \\
\text { prevenção de enfermidades e educação sanitária. O } \\
\text { farmacêutico responsável deverá comunicar a autoridade } \\
\text { sanitária local, detalhando as atividades que serão } \\
\text { desenvolvidas. }\end{array}$ \\
\hline $\begin{array}{l}\text { Perfil farmacoterapêutico e o } \\
\text { acompanhamento da } \\
\text { terapêutica farmacológica }\end{array}$ & $\begin{array}{l}\text { Atividade que permite ao farmacêutico identificar, } \\
\text { prevenir e solucionar problemas relacionados com a } \\
\text { terapêutica farmacológica. O profissional é o responsável } \\
\text { por estabelecer as prioridades a serem adotadas, entre } \\
\text { estas estão: características dos usuários, tipo de } \\
\text { enfermidade (s), características do(s) medicamento (s), } \\
\text { nomes dos prescritores e registro de reações adversas a } \\
\text { medicamentos. }\end{array}$ \\
\hline $\begin{array}{c}\text { Aplicação de medicamentos } \\
\text { injetáveis }\end{array}$ & $\begin{array}{l}\text { É um serviço que só poderá ser executado pelo } \\
\text { farmacêutico ou por profissional habilitado, mediante } \\
\text { prescrição do profissional habilitado. Os medicamentos } \\
\text { não deverão ser administrados se apresentarem alguma } \\
\text { alteração na cor, odor ou contenha corpo estranho. Após } \\
\text { a aplicação o farmacêutico deverá registrar, em livro } \\
\text { próprio. }\end{array}$ \\
\hline Ina & $\begin{array}{l}\text { É um serviço prestado pela farmácia, na qual deve ser } \\
\text { realizado mediante prescrição médica, devendo ser } \\
\text { registrada em um livro próprio. }\end{array}$ \\
\hline
\end{tabular}




\section{FONTE: CONSELHO FEDERAL DE FARMÁCIA, 2021.}

Segundo ÁLVARES (2009) os serviços farmacêuticos é um direito da sociedade e os proprietários que não são farmacêuticos, estão percebendo que esses serviços elevam a farmácia à condição de estabelecimento de saúde, pois os clientes são fidelizados pelos serviços prestados.

\subsection{IMPORTÂNCIA DO ACOMPANHAMENTO FARMACOTERAPÊUTICO AOS CLIENTES DA FARMÁCIA COMUNITÁRIA}

O acompanhamento farmacêutico na farmácia comunitária consiste, entre outras atribuições, o acolhimento da demanda ou queixa do paciente; a identificação das suas necessidades de saúde; a intervenção e a avaliação de resultados, bem como a documentação de todo o processo de cuidado (MOREIRA, 2013).

Muitos dos clientes que fazem uso de polimedicamentos têm dúvidas relacionadas à interação medicamentosa e alimentar. O aprazamento dos horários das tomadas das medicações deve ser feita pelo farmacêutico, durante a dispensação do medicamento para que haja um uso seguro e racional, diminuindo o risco de eventos adversos na atenção primária. Segundo a OMS (2013), pacientes que recebem AF diminuem $30 \%$ dos riscos supracitados. Os erros podem ser causados por fatores referentes à prescrição, dispensação, administração, consumo e no monitoramento da reconciliação medicamentosa (OMS, 2013).

De forma concomitante, o farmacêutico também deve evitar que problemas, advindos do uso dos remédios, sejam evitados ao máximo. Porém, esses efeitos indesejáveis ainda podem surgir e o profissional deve pensar numa solução. No caso da atenção farmacêutica, o farmacêutico pode atuar com outros profissionais, de forma multidisciplinar. Toda a equipe deve estar empenhada para que o paciente tenha o melhor aproveitamento possível advindo do uso da medicação (OMS, 2013).

Existem medicamentos que são dispensados sem a retenção de receita, ainda assim podem causar danos à saúde. Durante a AF faz-se necessário a conscientização da automedicação, a incompatibilidade com os medicamentos de uso crônico e o risco de intoxicação. O cliente deve ser orientado quanto à data de validade, forma de 
armazenamento e a demonstração da forma correta a ser utilizado (BRASIL, 2014).

Muitas farmácias comunitárias possuem postos de coleta de medicamentos vencidos e sem uso, sendo de responsabilidade do farmacêutico identificar e informar ao cliente sobre os postos de coleta afim de evitar que os resíduos das substâncias contaminem o meio ambiente (BRASIL, 2014).

\subsection{FARMÁCIAS COMUNITÁRIAS NO BRASIL}

No Brasil, a farmácia comunitária é o segmento profissional que reúne o maior número de farmacêuticos: aproximadamente $60 \%$ de todos os profissionais registrados nos Conselhos Regionais de Farmácia (SBFC, 2013). Entretanto, este segmento é talvez o que recebe a menor remuneração salarial, oferece menos perspectivas de crescimento e, assim, detém o maior índice de insatisfação profissional.

Segundo o Conselho Federal de Farmácia em seu relatório de atividades dos Conselhos Regionais de Farmácia (divulgado em dezembro de 2010), existem 82.204 estabelecimentos farmacêuticos no país, com a proporção de um estabelecimento para cada grupo de 3,2 mil pessoas, o que demonstra um número excessivo de farmácias no Brasil.

A situação atual das farmácias comunitárias no Brasil é de transformação. Os problemas são muitos, mas aponta-se um combate a eles. As ações clínicas na farmácia, antes restritas ao âmbito hospitalar, com o surgimento da atenção farmacêutica, expandem-se gradativamente para as farmácias comunitárias (MOREIRA, 2013).

Estas são incentivadas pelo novo engajamento dos profissionais farmacêuticos ancorados pelas instituições legais, modificações nas legislações e conscientização e cobrança da população. No Brasil, as farmácias e drogarias ainda estão distanciadas do seu papel sanitário. A dispensação de medicamentos nem sempre é entendida como processo de assistência à saúde; há insuficiência de orientação farmacêutica no momento da dispensação de medicamentos, tanto em estabelecimentos privados como nos públicos; e o profissional farmacêutico poucas vezes está presente nas 
farmácias para prestar adequadas informações e orientações (ROMANO-LIEBER; CUNHA; RIBEIRO, 2013).

Além disso, o farmacêutico não tem atuação destacada no acompanhamento da utilização de medicamentos, na prevenção e promoção da saúde e é pouco reconhecido como profissional de saúde tanto pela sociedade quanto pela equipe de saúde (IVAMA, 2013).

A revalorização do profissional farmacêutico e seu posicionamento diante de suas funções e importância no processo geral de saúde vêm sido enfatizadas e impulsionadas por mudanças que abrangem desde o ensino farmacêutico nas Universidades até mudanças importantes nas legislações e programas do Governo Federal. As Diretrizes Curriculares Nacionais do Curso de Graduação em Farmácia, aprovadas em 2002, abandonando os modelos tecnicistas e voltando-se para uma formação mais generalista, humanística e crítica, contribuiu, dessa forma, para a concepção de profissionais mais aptos a lidar com os desafios que o contexto atual exige (BRASIL, 2013).

O advento da Política Nacional de Medicamentos em 1998, dos medicamentos genéricos em 1999, (Lei 9.787/99), a criação do Sistema Nacional de Gerenciamento de Produtos Controlados - SNGPC (RDC 27/o7), a Resolução que dispõe sobre as Boas Práticas Farmacêuticas (RDC 44/o9), a criação dos NASF - Núcleos de Apoio à Saúde da Família e a e inclusão dos farmacêuticos nesses núcleos (RDC 154/o8), de projetos como Programa de Farmácias Notificadoras e Projeto de Fracionamento de Medicamentos, lançados pela Agência Nacional de Vigilância Sanitária (ANVISA), além de outros, tem contribuído, sobremaneira, para a revalorização do profissional farmacêutico (BRASIL, 2013).

A criação em abril de 2009 da Sociedade Brasileira de Farmácia Comunitária (SBFC), o curso de "Assistência Farmacêutica na Farmácia Comunitária” realizado pelo Conselho Federal de Farmácia (CFF) em várias capitais do Brasil, o aumento do número de fiscalizações (pelas Visas e Conselhos regional e federal) a fim de se verificar a qual idade dos serviços prestados e direitos dos farmacêuticos prestados e campanhas de conscientização da população sobre uso racional de medicamentos, 
sinalizam novos tempos para as farmácias comunitárias e de sua importância como centro propagador e extensor de saúde e não somente comerciais.

Resta ao farmacêutico buscar meio para se manter atualizado técnica e cientificamente, atuar na comunidade, unir-se aos colegas de profissão e às equipes de saúde multidisciplinares. A valorização do profissional parte do princípio da valorização pessoal.

\subsection{OBSTÁCULOS DA ATENÇÃO FARMACÊUTICA E O QUE PODE SER FEITO PARA INCENTIVAR SUA PRÁTICA}

A Atenção Farmacêutica no Brasil está sendo implantada aos poucos, principalmente pelo fato de que a maioria dos profissionais não tem uma formação voltada para tal, em muitos casos o profissional é forçado a lapidar-se com a prática diária e com as dificuldades encontradas no mercado de trabalho. Vale salientar a falta de cursos de especialização nesta área, ficando assim o profissional à margem do mercado de trabalho. Isto é bem ilustrado por um estudo realizado em Ribeirão Preto$\mathrm{SP}$, onde, dos treze farmacêuticos entrevistados, quase todos relataram ter obtido da graduação apenas uma base técnica e científica, ou ainda, como não tendo oferecido preparação nenhuma para o exercício da Atenção Farmacêutica (ARAÚJO FREITAS, 2013).

Os cursos de graduação em Farmácia precisam repensar seus projetos, priorizando o contato com pacientes e visualizando estes como pessoas inseridas em uma sociedade. Com isso, formar-se-iam profissionais com destrezas e habilidades para lidar com o medicamento que será utilizado pelo paciente (CORDEIRO LEITE, 2013).

O farmacêutico não deve utilizar seus conhecimentos apenas para dispensar medicamentos corretamente, mas também para realizar um acompanhamento farmacoterapêutico com qualidade. Avaliando os resultados obtidos pelas diferentes medicações, poderá detectar o possível aparecimento de efeitos adversos e, acima de tudo, acompanhar os objetivos terapêuticos almejados serão alcançados (KNOWLTON. 2013). 
Outro fato digno de relevância é a necessidade de reciclagem dos profissionais que já estão no mercado, por meio de iniciativas que contemplem a educação continuada (WHO, 2013).

A realização de eventos e a obtenção de fontes de atualização mais específicas sobre o trabalho assistencial farmacêutico constituem, ainda, necessidade dos profissionais entrevistados, assim como maior incentivo dos empregadores para a participação, dada a pouca disponibilidade de tempo e recursos financeiros relatados por eles (ARAÚJO FREITAS, 2013).

Além da falta de preparo dos profissionais, inúmeros outros obstáculos erguemse frente aos profissionais que objetivam a implantação da Atenção Farmacêutica, pois esta ainda é pouco conhecida e para que ela possa ser inserida como algo rotineiro nas farmácias brasileiras terá que superar resistências, provar seus benefícios e contar com profissionais que a divulguem. Na maioria das vezes, o farmacêutico da farmácia pública, hospitalar ou privada tem uma gama enorme de tarefas burocráticas que o afasta do paciente. Assim como ocorreu em outros países, o farmacêutico brasileiro precisa melhorar seu tempo, diminuindo as tarefas administrativas e aumentadas às atividades clínicas (BISSON, 2013).

Para uma melhoria efetiva no atendimento, as empresas deveriam dar incentivos para a atualização e especialização dos profissionais, fornecendo-lhes um tempo disponível para que este possa realizar cursos de atualizações, bem com incentivar, inclusive financeiramente, profissionais especializados, deste modo atribuindo o verdadeiro valor ao farmacêutico (ARAÚJO FREITAS, 2013).

Cabe ao farmacêutico buscar seu espaço e convencer os demais profissionais de sua importância neste novo modelo de prática profissional, a Atenção Farmacêutica, pois o estudo profundo desta prática torna-o unicamente qualificado para o acompanhamento terapêutico, já que possui amplo conhecimento sobre medicamentos e seus mecanismos de ação (CORDEIRO LEITE, 2013).

Para tanto seria necessária uma reestruturação das farmácias privadas, hospitalares e públicas, diminuindo as funções burocráticas executadas pelo farmacêutico, as quais tomam muito tempo e acarretam na diminuição do tempo 
dedicado ao atendimento e orientação dos pacientes, além das tarefas burocráticas, muitos farmacêuticos têm o dever de auxiliar na limpeza de prateleiras e organização das mesmas, o que agrava ainda mais a situação do atendimento (WHO, 2013).

\section{CONCLUSÃO}

As farmácias comunitárias não devem mais ser tratadas somente como estabelecimentos comerciais, mas sim, como um local de prestação de assistência farmacêutica e, de forma geral, assistência à saúde, caracterizando um local de promoção, proteção e recuperação da saúde, onde a atuação do profissional farmacêutico com embasamento clínico contribui diretamente na qualidade de vida da população e também para a diminuição na sobrecarga do sistema de saúde. Para esse fim, com o intuito de desempenhar suas atividades com total embasamento legal e científico, é de suma importância que o profissional farmacêutico esteja sempre e continuamente atualizando-se sobre as legislações vigentes e os avanços científicos na área para o cumprimento de suas funções com todo o respaldo legal necessário e garantindo a segurança e eficácia de suas ações à população.

As farmácias comunitárias devem adaptar-se em nível estrutural e profissional, com o intuito de atender uma demanda cada vez crescente de uma população necessitada e carente de serviços oferecidos com qualidade nos cuidados à saúde nesses estabelecimentos. Desta maneira para que a implantação da AF seja bem sucedida e contribua de modo eficiente para promover a saúde dos seus usuários é imprescindível o compromisso dos profissionais farmacêuticos aliado ao incentivo e adesão por parte dos proprietários destas farmácias e da população em geral.

\section{REFERÊNCIAS}

ARAÚJO AJA, FREITAS O. Concepções do profissional farmacêutico sobre a assistência farmacêutica na unidade básica de saúde: dificuldades e elementos para a mudança. Revista Brasileira de Ciências Farmacêuticas, v.42, n.I, jan./mar. 2013.

BARETA GMS. Pharmaceutical care in com-munity pharmacies of the city of Campina Grande do Sul. Visão Acadêmica. 2003. 
BASTOS, C. R.G.; CAETANO, R. As percepções dos farmacêuticos sobre seu trabalho nas farmácias comunitárias em uma região do estado do Rio de Janeiro. Ciência \& Saúde Coletiva. I5(Supl. 3), p. 3541-3550, 2010. Disponível em: http://www.scielo.br/pdf/csc/vi5s3/vi5s3a29.pdf. Acesso em: o2 de setembro de 202I.

BRASIL. Ministério da Saúde. Agência Nacional de Vigilância Sanitária. Lei no 5.991 , de 17 de dezembro de 1973. Dispõe sobre o controle sanitário do comércio de drogas, medicamentos, insumos farmacêuticos e correlatos, e dá outras providências. Brasília. Disponível em: http://www.anvisa.gov.br/legis/conso-lidada/lei_599I_73.htm. Acesso em 25 de agosto de 2021.

Brasil. Lei no 5.991, de 17 de dezembro de 1973. Dispõe sobre o controle sanitário do comércio de drogas, medicamentos, insumos farmacêuticos e correlatos, e dá outras providências. Diário Oficial da União, 1973.

BRASIL. Lei no 13021, de 08 de agosto de 2014. Dispões sobre o exercício e a fiscalização das atividades farmacêuticas. Publicada no D.O.U. de II de agosto de 2014. Subchefia para Assuntos Jurídicos, Brasília, DF, 2014.

BRASIL. Conselho Federal de Farmácia - CFF. RDC № 585, de 29 de agosto de 2013. Regulamenta as atribuições clínicas do farmacêutico e dá outras providências. Publicada no D.O.U. de 25 de setembro de 2013. Brasília, DF, 2013a.

BRASIL. Conselho Federal de Farmácia - CFF. RDC № 586, de 29 de agosto de 2013. Regula a prescrição farmacêutica e dá outras providências. Publicada no D.O.U. de 26 de setembro de 2013. Brasília, DF, 2013b.

BRASIL. Agência Nacional De Vigilância Sanitária. Resolução da Diretoria Colegiada n. 44 de 17 de agosto de 2009. Dispõe sobre Boas Práticas Farmacêuticas para o controle sanitário do funcionamento, da dispensação e da comercialização de produtos e da prestação de serviços farmacêuticos em farmácias e drogarias e dá outras providências. Diário Oficial União, Poder Executivo, Brasília, DF, I8 ago. 2013. Seção I, p. 78 .

CONSELHO REGIONAL DE FARMÁCIA DO ESTADO DE SÃO PAULO. Fascículo III Serviços farmacêuticos. São Paulo, SP, 2oro. Disponível em: http://portal.crfsp.org.br/phocadownload/fasciculo_iii_opas.pdf $>$. Acesso em: 02 de setembro de 202I.

CORDEIRO BC, Leite SN. O Farmacêutico na atenção à Saúde: conceitos, práticas e reflexões. 2.ed. Itajaí-SC: UNIVALI, 2013.

DOBLINSKI, P.M.F.; FORLIN,J.; FLORENCE, G. M. V.; MORANDI F; MELLO, J. C. P.; DELAPORTE R. H. Assistência e atenção farmacêutica: estudo comparativo entre dois bairros de classes sociais diferentes em Toledo -PR. Infarma ciências farmacêuticas. 2006. Disponível em: 
http://www.cff.org.br/sistemas/geral/revista/pdf/r2/info7air.pdf. Acesso em: o2 de setembro de 202I.

GALATO, D.; ALANO, G.M.; TRAUTHMAN, S.C.; VIEIRA, A.C. A dispensação de medicamentos: uma reflexão sobre o processo para prevenção, identificação e resolução de problemas relacionados à farmacoterapia. Revista Brasileira de Ciências Farmacêuticas, São Paulo, vol.44, n.3, p. 629 -640. Disponível em: http://www.scielo.br/scielo.php?script=sci_arttext\&pid=Si516-93322008000300017.

Acesso em: 04 de setembro de 202I.

IVAMA, A. M. et al. Consenso brasileiro de atenção farmacêutica: proposta. Brasília: Organização Pan-Americana da Saúde, 2013. Disponível em: http://bvsms.saude.gov.br/bvs/publicacoes/PropostaConsensoAtenfar.pdf Acesso em: 04 de setembro de 202I.

KNOWLTON CH, Penna RP. Pharmaceutical Care. New York: Hodder Arnold, 2013. p.243-256.

MOREIRA, D. F. F. Sistemas de distribuição de medicamentos: erros de medicação. Trabalho de Conclusão de Curso (especialização)-Escola de Saúde do Exército, Programa de Pós-Graduação em Aplicações Complementares às Ciências Militares. Rio de Janeiro, 2009.

ORGANIZAÇÃO MUNDIAL DA SAÚDE (OMS). Pharmacovigilance: ensuring the safe use of medicines. Policy Perspectives on Medicines, Genebra, v.9, p. I-6, out. 2013.

SOUZA, S. S. Farmacêuticos e suas atividades em farmácias comunitárias: uma análise de perfil. 2012. 90 p. Dissertação (Mestrado em ciências farmacêuticas) Centro de ciência da saúde, Universidade Federal do Rio Grande do Norte, Rio Grande do Norte, 2012. Disponível em: http://bdtd.bczm.ufrn.br/tde_arquivos/33/TDE-201303-04To14717Z-4856/Publico/SaralySS_DISSERT.pdf. Acesso em: 04 de setembro de 2021.

WORLD HEALTH ORGANIZATION. The Role of the Pharmacist in the Health Care System. Vancouver: WHO; 2013. 49 p. I6. Bisson MP. Farmácia Clínica e Atenção Farmacêutica. São Paulo: Medfarma, 2013. p.7-9. 